\title{
Pour une éducation sonore et musicale à l'école
}

\section{Sylvie Jahier}

\section{(2) OpenEdition}

\section{Journals}

\section{Édition électronique}

URL : http://journals.openedition.org/trema/1952

DOI : 10.4000/trema.1952

ISSN : 2107-0997

\section{Éditeur}

Faculté d'Éducation de l'université de Montpellier

\section{Édition imprimée}

Date de publication : 1 avril 1997

Pagination : 77-88

ISSN : 1167-315X

\section{Référence électronique}

Sylvie Jahier, « Pour une éducation sonore et musicale à l'école », Tréma [En ligne], 11 | 1997, mis en ligne le 01 avril 1997, consulté le 01 mai 2019. URL : http://journals.openedition.org/trema/1952 DOl : 10.4000/trema.1952

Ce document a été généré automatiquement le 1 mai 2019.

Trema 


\title{
Pour une éducation sonore et musicale à l'école
}

\author{
Sylvie Jahier
}

\section{Prélude}

1 Charlène, 5 ans, Moyenne Section de Maternelle, vient de faire entendre à ses camarades les sons qu'elle a trouvés en faisant glisser deux pots de yaourt en verre le long d'un mur recouvert d'une peinture granuleuse. L'institutrice lui demande de «faire vivre ses sons, de leur faire raconter quelque chose, de faire de la musique avec tout ça... Une musique qui nous fasse des surprises, avec un début et une fin. » Alors, Charlène commence par faire glisser doucement ses deux pots en décrivant de larges arabesques, puis elle accélère ses mouvements, s'arrête, reprend en intercalant des silences, produit des variations d'intensité et de vitesse, marque un temps d'arrêt un peu plus long que les autres. Pour terminer, elle lève les bras bien haut et fait redescendre ses deux pots le long du mur, puis bloque son geste à la fin de leur trajectoire. C'est seulement quand elle est arrivée au bout de ce geste qu'elle se retourne vers ses camarades, à qui elle tournait le dos pour jouer sur le mur. Dans cette courte séquence, mais si riche, Charlène a parcouru un trajet qui va d'une simple sensibilité sonore à une sensibilité incontestablement musicale. En effet, d'un jeu tâtonnant utilisant des sons découverts lors d'une phase d'exploration, elle aboutit à une production qui manifeste une intention de communiquer quelque chose à ses camarades, qui témoigne d'une maitrise de ses gestes et donc des sons qu'ils produisent, allant même jusqu'à les organiser en une forme structurée dans le temps.

2 Mathieu, même âge, même classe, joue avec ses mains sur les cordes d'un piano qu'on a débarrassé de son clavier, de ses marteaux, étouffoirs etc. Il alterne de grands balayages en frottant les cordes du grave à l'aigu, avec des coups frappés sur des cordes isolées. Il étouffe parfois la résonance, ou bien il la laisse au contraire se développer puis s'éteindre avant de produire un autre son. Il joue également en « dialogue » en faisant se répondre des sons graves et des sons aigus grâce au jeu en alternance de ses deux mains. Là encore, 
on assiste à la production d'un réel discours musical, dans lequel on peut distinguer plusieurs parties très différentes les unes des autres. L'enfant joue avec ces éléments du langage musical que sont les variations d'intensité, de vitesse, les silences, la résonance, les graves et les aigus etc. Les variations de ces sons sont produites par des variations des gestes exécutés par l'enfant, qui montre un engagement corporel complet dans son jeu et fait preuve d'une concentration soutenue.

3 Je pourrais ainsi, à travers les diverses expériences menées dans le cadre de mon travail de Conseillère Pédagogique en Education Musicale, multiplier les exemples où des enfants, de la PS de Maternelle au CM2, font preuve d'une grande sensibilité dans les domaines sonore et musical. Je pourrais parler de cette classe de CM2, d'une ZEP de la banlieue parisienne, ayant pratiqué diverses percussions tout au long de l'année. L'aboutissement du projet s'est manifesté à travers l'exécution collective d'une pièce musicale constituée de diverses mélodies, dont une comportant deux voix en contrechant, jouées sur des balafons et accompagnées de ponctuations et formules rythmiques. Les enfants jouaient sans aucune direction du maître et manifestaient un sens du rythme certain, car leurs différentes parties « s'encastraient » à la perfection, et respectaient avec un ensemble tout aussi parfait un changement de pulsation qui s'effectuait au milieu du morceau. Je pourrais parler aussi de ces classes d'écoles élémentaires qui avaient travaillé en finesse l'interprétation d'un répertoire de chants, dans lesquels le rapport entre texte et musique avait fait l'objet d'une analyse permettant de déterminer à quels endroits il serait judicieux de faire des nuances d'intensité, des accélérations, de tenir les sons ou au contraire de les faire très brefs etc. C'est toute la différence entre une classe qui chante de façon mécanique et identique tous les chants qu'elle a appris - ce qui est certes mieux qu'une classe qui ne chante pas du tout - et une classe qui a développé sa sensibilité, a compris qu'un chant est un message à délivrer, dont on peut accroître la musicalité, bref, en faire une interprétation « sensible ».

Je terminerai ici cette évocation, qui finirait par devenir fastidieuse, d'expériences et de projets divers menés dans le domaine musical et qui montrent à quel point les enfants de l'école maternelle et élémentaire peuvent faire preuve d'une sensibilité sonore et musicale. Il est possible, souhaitable, indispensable même de développer et d'enrichir cette sensibilité qui se manifeste spontanément en chaque être humain. La famille a un rôle à jouer dans ce domaine bien sûr, mais c'est ce qui peut et doit se faire à l'école qui nous intéresse en priorité ici, même si un détour par ce qui se passe "avant l'école » pourra fournir quelques éclairages.

5 Comment se manifeste cette sensibilité au monde sonore et musical? Quelles observations en a-t-on faites? De quelle façon les enfants cheminent-ils dans l'univers sonore, et développent-ils leurs facultés auditives et perceptives? Telles sont les questions auxquelles il faut tenter de répondre pour pouvoir définir les grands principes d'une éducation sensorielle, sensitive et musicale, et examiner ensuite quels problèmes pose la mise en œuvre de cette pratique éducative.

\section{Premier mouvement : Les comportements sonores spontanés}

6 Diverses études ont montré que, dans le ventre de sa mère, le fotus développe très précocement son ouïe. Bercé par le balancement dû aux mouvements de sa mère, 
notamment ceux de la marche, baignant dans le liquide amniotique, le foetus est assailli de stimulations auditives : bruits de respiration, de déglutition, de digestion de sa mère etc. Les bruits du monde extérieur lui parviennent filtrés par le liquide amniotique et tous les bruits internes. Il ne fait maintenant plus aucun doute que le bébé, à sa naissance, reconnait la voix de sa mère ainsi que celles des personnes de son entourage proche. De diverses expériences menées, je retiendrai la suivante : des mamans se sont engagées à chanter tout au long de leur grossesse, très régulièrement, une même chanson, simple de préférence. Quand l'enfant naît, on constate que lorsqu'il s'agite, pleure, manifeste quelque désagrément (autre bien sûr que causé par un réel problème de santé organique ou fonctionnel), si la mère lui chante cette même chanson, l'enfant se calme très vite, apparemment "rassuré» par ces retrouvailles avec cet élément sonore qui l'a accompagné tout au long de sa gestation.

7 Un peu plus tard dans la vie de l'enfant, des observations ont été menées en crèche, ${ }^{1}$ Là aussi, on constate que les enfants sont très attentifs aux événements sonores qui se produisent dans leur environnement. D'une part, l'enfant tourne la tête vers la source de ces événements sonores, que ce soit l'arrivée d'un chariot, la fermeture d'une porte etc. ou la manipulation d'un objet produisant des sons. D'autre part, l'enfant exploite spontanément les possibilités sonores des objets qui se trouvent à sa disposition, quitte à les détourner de leur fonction habituelle. Il exploite également spontanément les possibilités de sa voix, à travers divers gazouillis et premiers sons chantés. En outre, dans certaines crèches, des activités spécifiques d'éveil au monde sonore sont proposées aux enfants, notamment par le biais de la manipulation de divers objets et petits instruments. Contrairement aux idées reçues, on constate que les enfants sont capables de mobiliser leur attention sur des laps de temps tout à fait durables.

8 Ce qu'il faut absolument remarquer, et qui aura des conséquences sur le type d'éducation sonore à mener par la suite, c'est l'importance de l'action directe sur l'objet ou sur soimême, l'exercice de la motricité. L'enfant saisit progressivement le lien entre le geste effectué et le son produit par ce geste, et perçoit que c'est la variation du geste qui produit la variation du son. Un aller-retour se fait entre « je fais et j'écoute » et peu à peu c'est l'oreille qui va contrôler le geste. Celui-ci s'effectue progressivement avec une intention sonore, et non plus seulement motrice.

Sonorités chromatiques :

"I, pourpres, sang craché, rire des lèvres belles

Dans la colère ou les ivresses pénitentes ;

$U$, cycles, vibrements divins des mers virides,

Paix des pâtis semés d'animaux, paix des rides

Que l'alchimie imprime aux grands fronts studieux [...] "

A. RIMBAUD

Espérons que la famille sera attentive à toutes ces manifestations spontanées d'intérêt pour le sonore et proposera à l'enfant des situations de communication et de jeu passant par le son. On sait qu'un sens qui n'est pas stimulé peut s'émousser, et réduire une grande subtilité potentielle à une réalité perceptive d'une pauvreté navrante, et même «mutilante ». Mais que la famille joue ou non son rôle dans ce domaine, l'école a à sa disposition un champ d'action immense, permettant de développer chez tous les enfants leur perception auditive et leur sens musical. 


\section{Deuxième mouvement : Le sens musical}

10 A l'école maternelle, il est fréquent que l'enfant soit confronté à des activités portant sur la reconnaissance de sons, leur provenance etc. Du réveil caché que l'on doit retrouver grâce à son « tic-tac » aux divers lotos sonores portant sur les bruits de la vie quotidienne ou les bruits des éléments naturels, toutes activités qui développent l'oreille, mais qui ne portent pas encore sur ce qui est musical et ce qui ne l'est pas. Comment faire passer l'enfant d'une exploration sonore à une production musicale ? Quelle distinction opère-ton entre un comportement sonore et un comportement musical ?

11 Les représentations que l'on se fait de la musique, et de ce qu'est un musicien modèlent les réponses à ces questions. La représentation la plus communément répandue en France, aujourd'hui, est celle qui associe à «musicien » la pratique d'un instrument, de préférence appartenant à l'orchestre classique ou symphonique. Cette pratique s'apprend dans un conservatoire et passe par l'apprentissage du solfège. Le sens musical s'évalue à travers diverses capacités telles que: savoir déchiffrer une partition, percevoir un changement de tonalité dans une œuvre, distinguer une tonalité mineure d'une tonalité majeure, un accord mineur d'un accord majeur, savoir reconnaitre et chanter divers intervalles entre les notes, distinguer une pulsation binaire d'une pulsation ternaire, etc. Ces représentations sont très prégnantes. Les conséquences de leur pouvoir sont multiples. Socialement, on assiste à un clivage entre ce qui serait « la vraie musique », ce qu'on appelle la "grande » musique, et la musique diffusée le plus couramment par les médias : rock, variétés etc. Certaines musiques auraient plus de «valeur» que d'autres, même si ces dernières sont celles écoutées par le plus grand nombre. En outre, sont ignorées les musiques « savantes » contemporaines et la plupart des musiques ethniques même si on assiste à la promotion récente de musiques telles que les voix bulgares ou les chants corses.

De plus, en ce qui concerne l'éducation musicale à l'école, ces représentations produisent une double impossibilité : l'enseignant qui n'a pas reçu une culture musicale "classique » ne se sent pas capable d'y éveiller ses élèves. Il ne voit pas non plus comment exploiter les musiques du quotidien, celles des médias. Ce qui est cohérent d'ailleurs, car en dépit de la différence de style, les « codes » rythmiques et harmoniques sont les mêmes que ceux de la musique classique et symphonique. En conséquence, dans le meilleur des cas, il se contente de faire pratiquer le chant, à travers un répertoire spécifique de chansons pour enfants, et éventuellement conduit quelques activités rythmiques à base de frappés corporels, voire à l'aide de petites percussions présentes dans les écoles. L'écoute d'œuvres, quand elle est proposée, consiste le plus souvent à transcrire, à l'aide de dessins, de textes ou en s'exprimant corporellement, ce qu'évoque la musique écoutée. La musique proposée étant le plus souvent de type « musique à programme » : Le carnaval des animaux de St Saëns, Casse-Noisette de Tchaïkovski, ou de type « musique planante »: J.-M. Jarre, Vangelis etc. Mais ce que l'on fait travailler dans ce type d'activités est plus l'imagination visuelle et "littéraire», qu'une réelle approche de la nature du langage musical. 
Manuel scolaire de chant, fin du XIXe siècle.

BATAILLE F. (ancien instituteur, officier d'académie chargé de la classe primaire au lycée Michelet), Les Chansons de l'Ecole et de la Famille - Sur des airs populaires des provinces de France,

Arrangements à une, deux et trois voix, de ROUGNON P., $2^{\mathrm{e}}$ édition, revue et corrigée, Paris, Éd. Classique Eugène Belin, 1891, page 48.

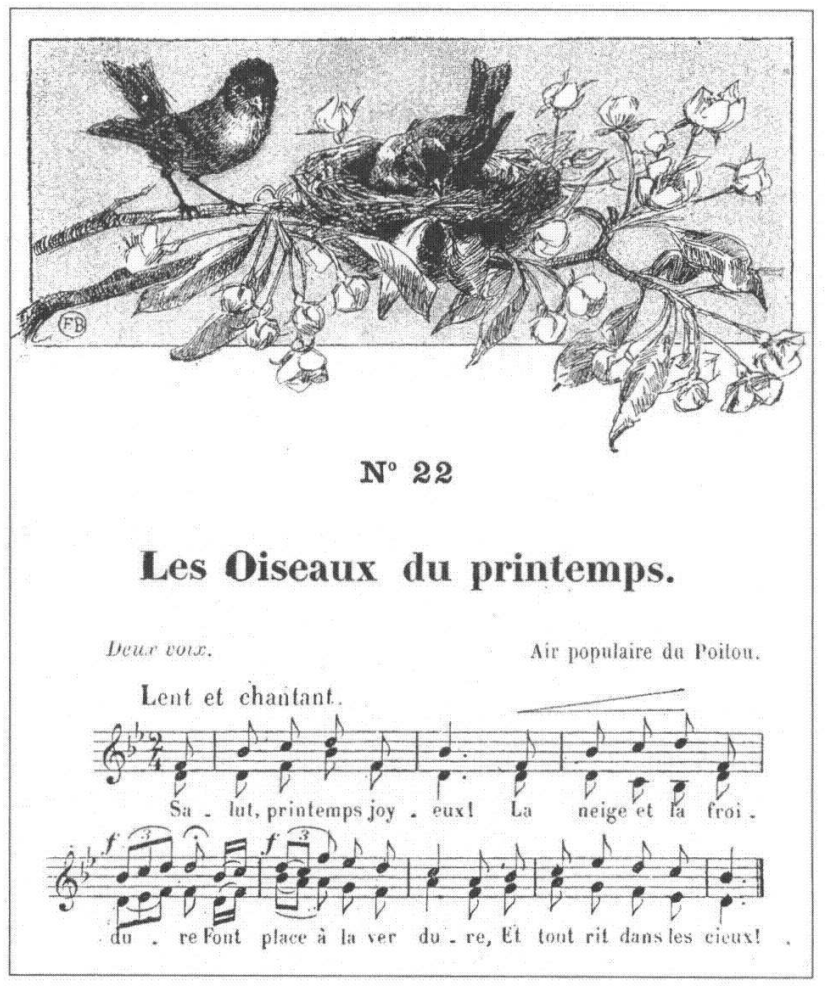

Il est d'autres façons d'aborder une œuvre musicale, quelle qu'elle soit : par quels moyens le musicien s'exprime-t-il dans ses compositions ou ses interprétations? Quels éléments $\mathrm{du}$ langage musical utilise-t-il ? Comment les combine-t-il ? Toutes ces questions permettent de développer une écoute active, une écoute qui interroge, plutôt que de se servir de l'œuvre comme inducteur d'un autre moyen d'expression. Elles ne nécessitent pas de connaître d'une façon approfondie toutes les règles de composition, les règles d'harmonie etc. mais nécessitent de prendre en compte la manière dont les enfants cheminent dans cet univers du sonore et du musical.

\section{Troisième mouvement : Bousculer les représentations...}

14 Nous avons vu plus haut que les enfants entrent dans le monde sonore par le biais de l'activité motrice. Que l'action et l'écoute sont intrinsèquement liées, qu'elles se développent l'une à travers l'autre. Si l'on cesse d'analyser la musique à travers son écriture (la plupart des musiques de notre planète ne sont d'ailleurs pas écrites), et que l'on observe ce qui se passe quand un musicien joue, on découvre que son «comportement» a beaucoup de points communs avec le comportement sonore spontané de l'enfant. François Delalande ${ }^{2}$ nous permettra de préciser tout cela. Pour lui, peu importe de ne pouvoir définir la musique, tant elle se présente sous des formes différentes selon les lieux et les époques. En revanche, il importe de définir ce qu'est un 
«musicien»: Quelle que soit la culture, le style, l'époque d'un musicien, il fait preuve d'un certain nombre d'aptitudes qui constituent le « dénominateur commun » à toutes les pratiques musicales :

- A un premier niveau d'analyse, le musicien fait preuve d'un « [...] goût pour le son [...], » d'« une certaine sensualité de la sonorité qui s'accompagne de l'habileté à l'obtenir sur son instrument. $»^{3}$

- A un deuxième niveau d'analyse, le musicien est quelqu'un pour qui « [...] les sons prennent un sens, peuvent évoquer des états affectifs ou avoir une valeur symbolique dans sa société ou bien donner naissance à des images. Bref, sous une forme ou sous une autre, il y a une dimension imaginaire de la musique à laquelle il faut être sensible, sans quoi la musique à proprement parler, ne vous dit rien $»^{4}$

- Enfin, à un troisième niveau d'analyse, un musicien est sensible aux organisations des sons et apte à les produire.

De cette «nouvelle » définition, plus large, il ressort que l'éducation musicale ne vise pas l'acquisition de savoirs «sur » la musique, ni ne se réduit à l'acquisition de compétences strictement liées à un type de musique spécifique (par exemple: chanter juste, savoir reproduire des formules rythmiques mesurées, avoir des notions de solfège "classique " etc.). Il s'agit d'aider l'enfant à développer des conduites musicales, ces conduites étant liées à la définition du «musicien» et correspondant, en outre, aux trois stades du jeu chez Piaget :

1. Le stade sensori-moteur: quand le musicien, qu'il soit chanteur, violoniste, flûtiste etc. produit un son, une suite de sons ou une phrase musicale, il met en œuvre en premier lieu une action motrice. Les gestes produits lui servent à «modeler » les sons, à les rendre expressifs. Le fait même de produire ces gestes fait l'objet de sensations de plaisir ou de tension, et ces sensations sont (ou devraient être) en relation constante avec les sensations reçues par l'oreille, les unes et les autres s'influençant et se corrigeant mutuellement. Il en est de même pour l'enfant qui adopte une conduite musicale, et on doit pouvoir repérer ce plaisir procuré par l'exercice sensori-moteur.

2. Le stade symbolique: Une des fonctions de la musique est d'exprimer des sentiments. En outre, «[...] la musique peut évoquer des images visuelles, mais aussi déterminer chez l'auditeur des associations indépendantes d'une quelconque imitation du réel par les formes sonores. On peut donc parler d'une fonction symbolique de la musique à condition de nommer par cette expression l'existence d'un renvoi à une réalité absente sans affirmer qu'il s'explique exclusivement par un symbolisme imitatif $»{ }^{5}$ Certaines conduites musicales des enfants peuvent donc avoir cette dimension symbolique, comparable à celle qu'ils intègrent dans d'autres formes de jeux sans lien avec le monde sonore, (cf. ill. p. 80).

3. Le stade du jeu à règles: dans la plupart des pratiques musicales existe un «jeu combinatoire». En ce qui concerne la musique occidentale, la composition, l'écriture ou l'invention d'une mélodie apparaissent bien comme « un agencement d'unités non pas selon le caprice ou le hasard, mais selon des règles $» .^{6}$

Il semble donc qu'une éducation musicale adaptée à l'enfant passe par la mise en œuvre d'activités où vont s'imbriquer activités de production sonore et activités plus spécifiques d'écoute, même s'il est entendu que les activités de production sonore sollicitent l'écoute de ce que l'on fait ou de ce que font les camarades. Des manipulations d'objets, d'instruments, l'exploration de leurs possibilités sonores, l'exploration des possibilités sonores de la voix permettront de faire découvrir divers caractères des sons : il y a des sons « qui brillent », des sons « lisses » ou « rugueux », des sons « clairs » ou « sombres ». 
On peut faire varier l'intensité de ces sons, leur vitesse d'émission, leur rythme de succession. Certains d'entre eux résonnent, d'autres pas etc.

Passée cette première phase, on attire l'attention des enfants sur la valeur symbolique des sons que l'on produit et sur l'intention que l'on se donne: que peut-on raconter, sans mots, avec ces sons, pour exprimer un sentiment, pour communiquer quelque chose à ses camarades. Veut-on leur faire peur? Les apaiser? Les faire danser? Les faire rire? En outre, attirer l'attention de l'enfant sur les différentes façons de combiner ses sons, sur l'élaboration de différentes "parties" dans ce qu'il joue, lui permet d'une part de prendre du recul par rapport à ses impulsions, d'autre part de «relancer» ses idées, de les développer. Préciser à l'enfant que sa «musique» doit avoir une fin lui permet de prendre conscience que l'écoulement du temps est un élément structurant de la musique. Si c'est une production à plusieurs, préciser qu'il doit écouter ses camarades, tenir compte de ce qu'ils jouent, permet à l'enfant de prendre conscience que des sons peuvent se répondre, se superposer, s'accumuler etc. Tous processus qu'utilisent les musiciens, et qu'il sera plus facile d'entendre car on les aura expérimentés auparavant, qu'on aura été soi-même confronté à la difficulté, mais aussi au plaisir de mettre en forme les trouvailles réalisées au cours de la phase de tâtonnement. " Ecouter pour faire, faire pour écouter ", tel est le principe de base développé par M. Frapat.7 On peut procéder de deux manières : soit l'enseignant propose un matériau à explorer, dont on organise les trouvailles, et fait ensuite entendre une œuvre dans laquelle sont utilisés des modes de jeux ou des principes de composition qu'ont utilisés les enfants; soit l'enseignant fait entendre une œuvre et propose une activité de production sonore en lien avec ce que l'on a entendu.

On peut trouver dans le coffret "L'éducation artistique ${ }^{8}{ }^{8}$ élaboré par la Direction des Ecoles du Ministère de l'Education Nationale et diffusé dans toutes les écoles en 1994, des suggestions d'activités et d'œuvres à écouter, selon cette démarche. Ainsi, "Mâât", d'Henri Fourès, compositeur contemporain, sert de point de départ à une production vocale. Les enfants tentent d'imiter avec leur voix des sons présents dans l'œuvre. Ils constatent que ces sons sont organisés selon deux principes : la répétition d'un même élément et la superposition de différents plans sonores. A partir de cette première imitation, place est laissée à l'improvisation, à la recherche d'autres sons, à l'élaboration d'une " pièce musicale " propre à la classe, reprenant les deux principes de composition énoncés ci-dessus. Plus tard, on écoutera une fugue de J.S. Bach et l'on s'apercevra qu'il utilise, lui aussi, la répétition (de phrases mélodiques) et leur superposition (polyphonie). On écoutera une musique africaine où ces éléments sont présents également, mais de laquelle on extraira une cellule rythmique, la pulsation etc. qui serviront de points de départ à une nouvelle production d'un autre style. Ainsi peut-on cheminer tout au long de l'année en développant sa sensibilité musicale par la façon dont on affine les sons et les formes sonores qu'on produit, et par la façon dont on aiguise son oreille en cherchant à entendre « comment sont fabriquées » les œuvres qu'on écoute.

\section{Coda : que la musique retrouve sa place à l'école !}

Le Nouveau Contrat pour l'Ecole, dont l'application a débuté cette année, préconise une pratique quotidienne de la musique à l'école (mesure $\mathrm{N}^{\circ} 9$ ). Les programmes et instructions officielles ont été réorganisés et mis en relation avec les compétences à atteindre pour chaque cycle. L'éducation musicale, selon ces textes, se comprend par la 
mise en œuvre de trois types d'activités complémentaires: activités vocales, activités corporelles et instrumentales, activités d'écoute. Par exemple, au cycle 2 :

- pratique régulière du chant pour stabiliser la voix, rechercher la justesse, contrôler la respiration et parfaire l'articulation;

- jeux vocaux, à des fins expressives, pour les réinvestir dans des situations telles que la sonorisation de contes;

- participation à des activités instrumentales en recherchant une plus grande habileté gestuelle;

- écoute et identification de sons divers, repérage de leurs caractéristiques (intensité, timbre, hauteur, durée).

On constate que les activités préconisées sont de deux ordres : des activités à caractère «technique », comme par exemple: développer le sens rythmique et mélodique c'est-àdire développer la justesse et la rigueur de la reproduction par rapport au modèle entendu, et des activités plus ludiques, laissant la place à l'improvisation et l'invention, et faisant plus directement appel à la « sensibilité ». Développer la technique et développer la sensibilité ne sont pas contradictoires : la sensibilité qui sert l'expressivité utilise les acquis de la technique, et le développement de la technique prend son sens par son réinvestissement dans les activités de création et d'invention. Seul le travail technique gratuit (apprentissage du solfège dissocié de l'apprentissage d'un instrument par exemple) peut paraître desséchant, fastidieux et ingrat.

21 La simple lecture de ces programmes répond à la question « Que faire en musique avec ses élèves ? ». De multiples ouvrages fournissent des idées à foison ainsi par exemple, le CRDP de Bourgogne vient de publier un coffret pédagogique ${ }^{9}$ proposant des fiches pédagogiques extrêmement riches et simples d'emploi. Mais savoir "quoi faire » avec ses élèves ne délivre pas de la question "comment faire?", ni plus subtilement "qui être avec ses élèves quand on s'aventure dans ce domaine si vaste, et trop souvent méconnu, qu'est le monde musical? » Le pédagogue est avant tout une personne. Doté des meilleurs outils pédagogiques, c'est toujours son être profond qui entre en relation avec les élèves, et cette relation se construit à partir de l'attitude générale et de l'ensemble des valeurs qu'il a adoptés. Peut-on aider les enfants à développer leur imagination, leur créativité, leur sensibilité si on ne cherche pas à les développer soi-même?

Quand on interroge les maîtres sur les raisons pour lesquelles ils délaissent le plus souvent l'éducation musicale, la réponse unanime dénonce le "manque de formation ». Certes, un stage de formation apporte une expérience irremplaçable. C'est l'occasion de redécouvrir ses facultés de production, d'invention, d'improvisation. L'occasion aussi de (re)découvrir des œuvres variées, de mieux les entendre et mieux les comprendre, ce qui revient au même. Ne dit-on pas «j'entends bien » à quelqu'un à qui l'on veut assurer qu'on l'a compris? Mais les effets d'un stage s'émoussent vite si on ne cultive pas ce que l'on y a découvert, appris, expérimenté. C'est une question d'entrânement et de confiance, de plaisir aussi. Même s'il est vrai que dans le parcours professionnel de beaucoup d'enseignants, le nombre d'heures de formation dans le domaine musical est souvent insuffisant, gardons à l'esprit que la formation ne peut pas tout... qu'on a bien souvent le sentiment de ne jamais en avoir assez... que la demande de formation fait partie de ces demandes à caractère fantasmatique dont la nature même est de ne jamais pouvoir être assouvies!

Dans le domaine musical, encore moins que dans les autres disciplines enseignées à l'école, il ne s'agit pas de "posséder » un savoir, que l'on se hasarderait à « transmettre » une fois qu'on pense l'avoir suffisamment maîtrisé. Le maître peut et doit cheminer avec 
les enfants, découvrir en même temps qu'eux certaines finesses du monde sonore et musical. S'il ne se sent pas sûr de lui, qu'il se laisse guider par les enfants, et surtout qu'il leur fasse confiance. Ils ont beaucoup à nous apprendre, nous les adultes, qui avons parfois refermé les portes de nos sens... Laissons vivre en nous l'enfant qui vivait toutes oreilles ouvertes...

\section{NOTES}

1. BUSTARETA., L'oreille tendre, Paris, Éditions ouvrières.

2. DELALANDE F., La musique est un jeu d'enfant, Paris, Éd. Buchet-Chastel.

3. DELALANDE F, op. cit.

4. FRANCÉS R., La perception de la musique, Paris, Vrin. Cité par Delalande, in op. cit.

5. FRANCÉS R., op. cit.

6. FRANCÉS R., op. cit.

7. BEN HAMMOU A, CLÉMENT G. \& FRAPAT M., L'oreille en colimaçon, Paris, Éd. Armand Colin.

8. L'éducation artistique à l'école, Paris, Ministère de l'Education Nationale, Direction des Ecoles, Collection «une école pour l'enfant, des outils pour le maître», CNDP, Diffusion Hachette.

9. BACHELARD A., COULON D. \& LOISY J.-P., La musique au quotidien au cycle 2, parcours d'apprentissages de la maternelle au CE1, Dijon, CRDP de Bourgogne.

\section{RÉSUMÉS}

Comment le dispositif scolaire prend-il en compte la sensibilité au monde sonore et musical des enfants, de la petite section de maternelle jusqu'au CM2. De quelles façons cheminent-ils dans cet univers et développent-ils leurs facultés auditives et perceptives? Telles sont les questions auxquelles tente de répondre une Conseillère Pédagogique en Education Musicale en s'appuyant sur les pratiques les plus innovantes, inspirées des travaux du Groupe de Recherche Musicale (G.R.M) et d'une définition de la musique et du musicien qui se centre sur l'activité et la sensualité des sons, ainsi que l'habileté à les produire sur son instrument, quel que soit ce dernier.

How does the school system take into account the children 's sensibility to the world of sound and music from the first years of nursery school to the last year of primary school? Hox do they make their way in the universe and develop their eyes and ears? These are the questions which a "Conseillère pédagogique en Education Musicale" is trying to answer basing her arguments on the most up-to-date practices inspired by the research work clone by the "Groupe de Recherche Musicale" (G.R.M) and on a definition of music and the musician centred upon practice and the sensuality of the rounds, as well as the skill at producing them on an instrument, whichever it may be. 
INDEX

Keywords : child, ear, musical education, sensibility

Mots-clés : éducation musicale, enfant, oreille, sensibilité

\section{AUTEUR}

\section{SYLVIE JAHIER}

C.P. en éducation musicale, académie de Paris 